\title{
SEGREGATION OF STIMULUS PHASE AND INTENSITY CODING IN THE COCHLEAR NUCLEUS OF THE BARN OWL ${ }^{1}$
}

\author{
W. E. SULLIVAN ${ }^{2}$ AND M. KONISHI \\ Division of Biology, California Institute of Technology, Pasadena, California 91125
}

Received October 7, 1983; Revised January 16, 1984; Accepted January 20, 1984

\begin{abstract}
The cochlear nucleus of the barn owl is composed of two anatomically distinct subnuclei, $n$. magnocellularis (the magnocellular nucleus) and $\mathrm{n}$. angularis (the angular nucleus). In the magnocellular nucleus, neurons tend to respond at a particular phase of a stimulus sine wave. Phase locking was observed for frequencies up to $9.0 \mathrm{kHz}$. The intensity=spike count functions of magnocellular units are characterized by high rates of spontaneous activity, a narrow range of intensities over which spike counts changed from spontaneous to saturation levelé, and a small increase in spike counts with intensity over that range. In the angular nucleus, neurons showed little or no tendency to respond at a certain sinusoidal phase, although some showed weak phase locking for frequencies below $3.5 \mathrm{kHz}$. Angular units typically had low spontaneous rates, large dynamic ranges, and large increases in spike counts with intensity, resulting in high saturation levels. The clear difference between the two nuclei in sensitivity to both phase and intensity and the reciprocity in response properties support the hypothesis that each nucleus is specialized to process one parameter (phase or intensity) and not the other.
\end{abstract}

The mammalian cochlear nucleus is traditionally divided into three major divisions, the anteroventral, posteroventral, and dorsal cochlear nuclei (Ramón y Cajal, 1909; Lorente de No, 1933; Rose et al., 1959; Osen, 1969). Each major division presumably contains several subdivisions (Brawer et al., 1974). The cochlear nucleus of birds consists of two anatomically distinct nuclei, $n$. angularis and $\mathrm{n}$. magnocellularis (hence referred to as the angular and magnocellular nuclei). In both birds and mammals, each individual cochlear nucleus contains a complete representation of the audible frequency range. The existence of multiple cochlear nuclei implies that each must have a separate function. What those functions may be remains unresolved.

We studied the physiological response properties of neurons in the angular and magnocellular nuclei of the barn owl (Tyto alba) to determine what roles they might have in sound localization. Behavioral studies have shown that barn owls localize sounds in two dimensions by using a different interaural cue for each dimension-

\footnotetext{
${ }^{1}$ We thank M. Walsh and D. Hodge for designing and building some of our electronic equipment. Drs. T. Takahashi, A. Moiseff, and E. Knudsen provided helpful comments during the course of the experiments and on the manuscript. The work was supported by National Institutes of Health Grant NS 14617-04 to M. K. and National Institutes of Health Postdoctoral Fellowship NS 07045-01 to W. S.

${ }^{2}$ To whom correspondence should be addressed.
}

interaural phase differences for azimuth and interaural intensity differences for elevation (Knudsen and Konishi, 1979; Moiseff and Konishi, 1981). Physiological investigations of binaural neurons in the medulla and pons showed that these neurons were sensitive to either interaural phase or interaural intensity differences but not to both (Moiseff and Konishi, 1983). This suggested a functional separation of phase and intensity processing which might originate in the two cochlear nuclei. Therefore, we examined the sensitivity of neurons in each nucleus to stimulus phase and intensity. Our results show that there is a clear functional distinction between the two cochlear nuclei in the processing of these parameters.

\section{Materials and Methods}

Barn owls were anesthetized with Ketamine (4 mg/ $\mathrm{kg} / \mathrm{hr}$ ). The birds were placed in a stereotaxic head holder, and a small steel plate was mounted to the skull with dental cement to allow the head to be rigidly fixed during physiological recording. The skull overlying the cerebellum was removed. In some experiments, the cerebellum was partially removed by aspiration to expose the cochlear nucleus for direct electrode placement. In other experiments, the cerebellum was left intact, and stereotaxic coordinates were used to guide electrode placement. The experiments were conducted in a large sound-attenuated, anechoic chamber. The birds were 
placed on a heating pad to maintain a normal body temperature $\left(40^{\circ} \mathrm{C}\right)$ during the experiment.

Glass-coated platinum-iridium electrodes were used for single unit recordings. The electrodes were advanced by a remote controlled stepping motor. Neural signals were amplified by a custom-made pre-amp/Amplifier and displayed on a Tektronix 5110 oscilloscope. The output of the vertical amplifier of the oscilloscope was fed to an Ortec window discriminator. In some experiments a custom-made peak time detector was used to provide spike discrimination. The output pulses from the spike discriminator provided the input to a PDP 11/40 computer, which was used for data collection and analysis. An audio monitor (Grass AM8) was used which could be set to broadcast either the output of the electrode amplifier or the pulse output of the discriminator.

Acoustic stimuli in the form of noise or pure tone bursts were delivered at a rate of 1.5 stimuli/sec through earphones placed in the external auditory canals. Sine waves were generated by either a General Radio 1310-B oscillator or by a computer-controlled oscillator. Sinusoidal or wide band noise signals were shaped by an electronic switch to produce sound bursts. Typically, these were $100 \mathrm{msec}$ in duration and had rise/decay times of $5 \mathrm{msec}$. The signal was then attenuated by a HewlettPackard HP 350D manual decade attenuator in series with a computer-controlled attenuator, so that intensity could either be under manual or program control.

For the study of phase coding, period histograms were obtained. A positive zero-crossing detector was used to detect the beginning of each sine wave cycle. The zerocrossing pulses activated a computer-controlled clock which began counting from zero at each pulse. When a spike was detected, the current clock value was stored in a buffer. By recording over a large number of stimulus cycles, a list of spike times relative to the onset of the sine wave period could be collected. The spike times were normalized to the period of the stimulus (reciprocal of the frequency) and collated to form a period histogram in which the length of each bin represents the number of spikes which occurred at a particular phase of the sine wave cycle.

Vector strength analysis was used to quantify the period histograms (Goldberg and Brown, 1969). Vector strength was calculated by assigning each histogram bin a vector angle (based on the position of the bin) and length (the number of spikes in the bin) and adding all bins vectorally. The angle of the summed vector represents the mean phase of the response. The length of the summed vector is normalized to total spike counts so that a value of 1.0 is obtained if all spikes fall within one bin and a value of 0.0 is obtained if the spikes are evenly distributed throughout the bins.

For the study of intensity coding, the response to 100 msec tone bursts was recorded for various intensities. The computer randomized the order of intensities, and for each intensity of stimulation, the computer collected spike counts per stimulus, peristimulus time (PST), and interval histogram data. The PST histograms were used to calculate average response latency as a function of intensity. The interval histogram provided information on the average rate of discharge (the reciprocal of the mean firing interval).

\section{Results}

\section{Sensitivity to stimulus phase}

Phase locking in the magnocellular nucleus. We recorded from a total of 286 magnocellular neurons. The electrodes were placed on or near the midline of the medulla to record from fiber tracts containing magnocellular axons. Magnocellular units responded most often at a particular phase of a sinusoidal stimulus. In other words, their response was phase-locked. We observed phase-locked responses at frequencies up to about 9.0 $\mathrm{kHz}$. Figure 1 shows examples of period histograms (response versus stimulus phase) for four magnocellular neurons responding to $3-, 5-, 7-$, and $9-\mathrm{kHz}$ stimuli. Of the 286 units isolated, 268 of these showed clear phase locking $(93.7 \%)$. Nearly all of the neurons which did not display phase-locked responses (17 of 18) had best frequencies from 7 to $9 \mathrm{kHz}$, but these accounted for only $13.6 \%$ of the neurons sensitive to frequencies in this range.

Vector strength as a function of a unit's best frequency is plotted for the magnocellular population in Figure 2. (The value chosen for a given unit was the value for the response at best frequency and $20 \mathrm{~dB}$ above threshold.) It shows that the degree of phase locking decreases with increasing frequency. We have observed this phenomenon both with spike detectors which measure the time of occurrence of the action potential peak and the time at which a fixed voltage level is crossed. Tests with artificial spikes and mathematical simulations show that peak time detection is much less susceptible to fluctuations in spike time measurement due to the effects of random noise. Nevertheless, the two methods gave fairly equivalent results.

Mean phase changes with frequency. The effect of small changes in stimulus frequency on the mean phase of a unit's response (equivalent to the position of the histogram peak) was examined in 179 units. An example of such an experiment is shown in Figure 3. In this case, as in every other, an increase in stimulus frequency is associated with a dclay in the mean response phase.

This change in mean phase of response with frequency is due to the fact that in constructing a period histogram, spike times are measured with respect to the sine wave period present at the time the spike is detected, not to the one which caused the neuron to respond. The time difference between the activating and measurement sine wave periods corresponds to the neuron's response latency, which is about 2.5 to $3.0 \mathrm{msec}$ for magnocellular neurons. The response latency equals the sine wave period length ( 1 /frequency) times the number of periods (integer + fractional) between activation and measurement. A change in period length causes the reciprocal change in period number. If the change in period length is small enough, only the fractional portion of the number of periods will be affected, and a phase shift will result. The latency can be calculated by measuring the rate of change of phase with frequency. When the response latency was directly measured in a PST histogram, there was always a good agreement between this measurement and the value calculated on the basis of phase shifts (see Fig. $3, B$ and $C$ ).

This dependence of mean response phase on stimulus 

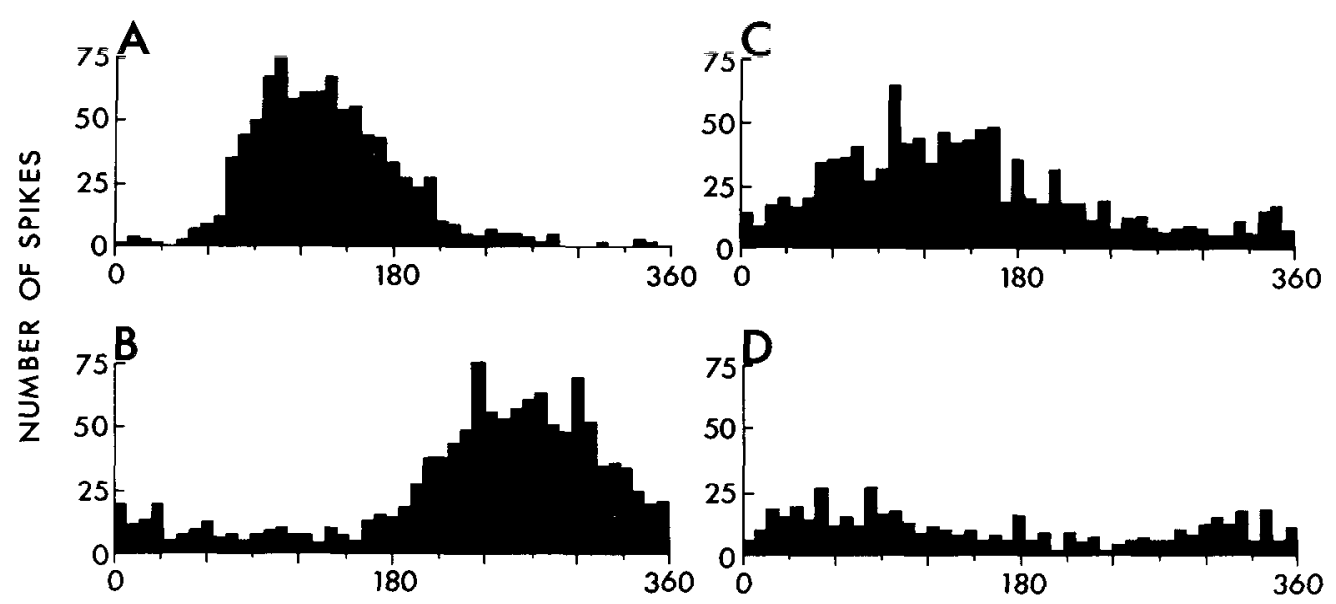

STIMULUS PHASE IN DEGREES

Figure 1. Period histograms for the response of four magnocellular neurons. A, Unit 14015$\mathrm{V}$; frequency $=3105 \mathrm{~Hz}$; amplitude $=64 \mathrm{~dB}$ SPL (re 0.0002 dyne $/ \mathrm{cm}^{2}$ ); 933 spikes in 10,000 periods; vector strength $(r)=0.714$. $B$, Unit $13383-\mathrm{A}$; frequency $=5208 \mathrm{~Hz}$; amplitude $=64$ $\mathrm{dB}$ SPL; 1178 spikes in 15,000 periods; $r=0.507$. $C$, Unit 13324-A; frequency $=7024 \mathrm{~Hz}$; amplitude $=64 \mathrm{~dB}$ SPL; 1030 spikes in 20,000 periods; $r=0.403 . D$, Unit 3316-B; frequency $=8928 \mathrm{~Hz}$; amplitude $=74 \mathrm{~dB}$ SPL; 486 spikes in 10,000 periods; $r=0.240$.

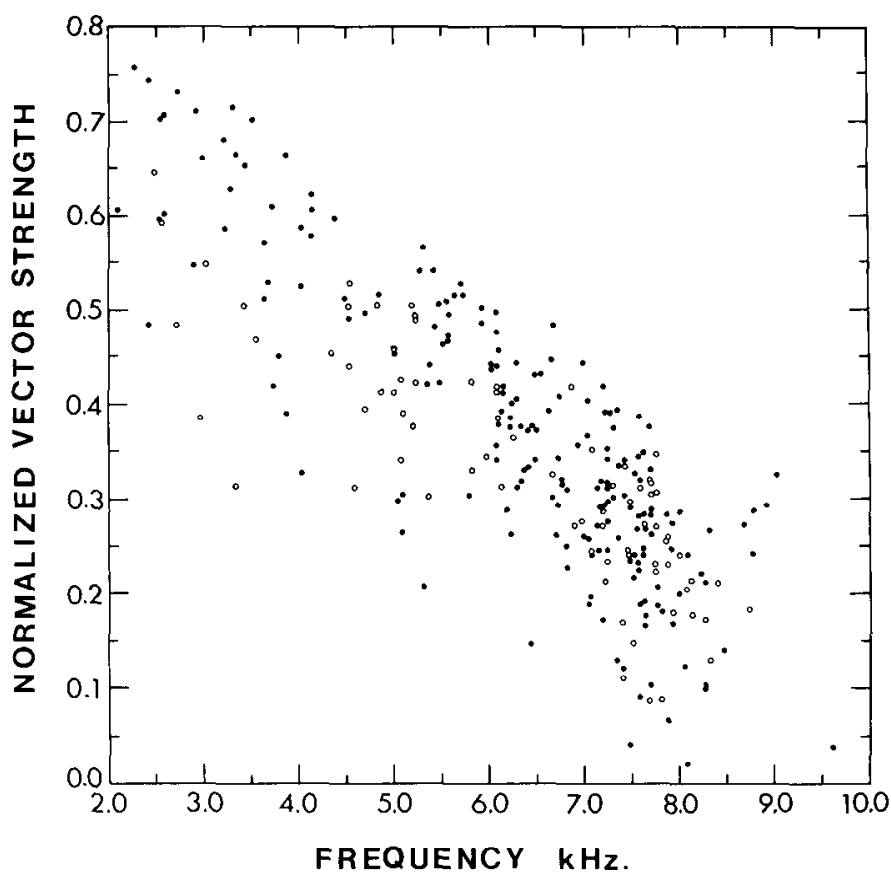

Figure 2. Vector strength as a function of best frequency for 286 magnocellular neurons. Solid circles, level detection used to discriminate spike arrival times. Open circles, peak-time detection used for spike time measurement.

frequency can be used to eliminate the possibility that phase locking could be due to an electrical artifact. Addition of the stimulus sinusoidal signal into the neural recording (due to pickup of the earphone's electromagnetic field by the high impedance recording electrode) can cause the appearance of phase locking when level detection is used to measure spike times (Johnson, 1978). During the positive phase of the sinusoidal modulation the spike reaches threshold faster than normal, and during the negative phase the spike is delayed. At higher frequencies, the slope of the sinusoidal noise is greater, and the time difference between positive and negative phases is smaller. These combine to increase the likelihood of artifactual phase locking. However, because the interaction between the sinusoidal noise and the spike occurs at the time the spike is detected, changes in period length should cause little or no change in the mean phase of the response. When we deliberately introduced a strong artifact into the recording, we were able to confirm this prediction. This shows that our results for phase locking at high frequencies cannot be due to an electrical artifact but must be physiologically related.

While sinusoidal noise can cause false positive results, random noise can cause false negative results. Improved single unit isolation often uncovered some phase locking where previously there had been none. This was especially true for high frequency neurons. In cases where there is a weak modulation of the period histogram, the criterion of a positive phase shift with increased frequency can be used to discern physiologically related phase locking. This method is superior to other methods of discrimination which select some arbitrary cutoff level (along some quantitative measure of phase locking such as vector strength) and reject any responses which fall below that level. These methods are artificial because some assumption must be made about what does or does not represent a level of information which is meaningful to the nervous system.

Effects of stimulus intensity on phase coding. We investigated the effects of changes in stimulus intensity on the phase-locked response of 28 magnocellular units. This was usually done at frequencies below, near, and above the best frequency of the unit. The data from these units were consistent in the following respects: (1) At the unit's best frequency, changes in intensity had no effect on the mean phase of the response. (2) For fre- 


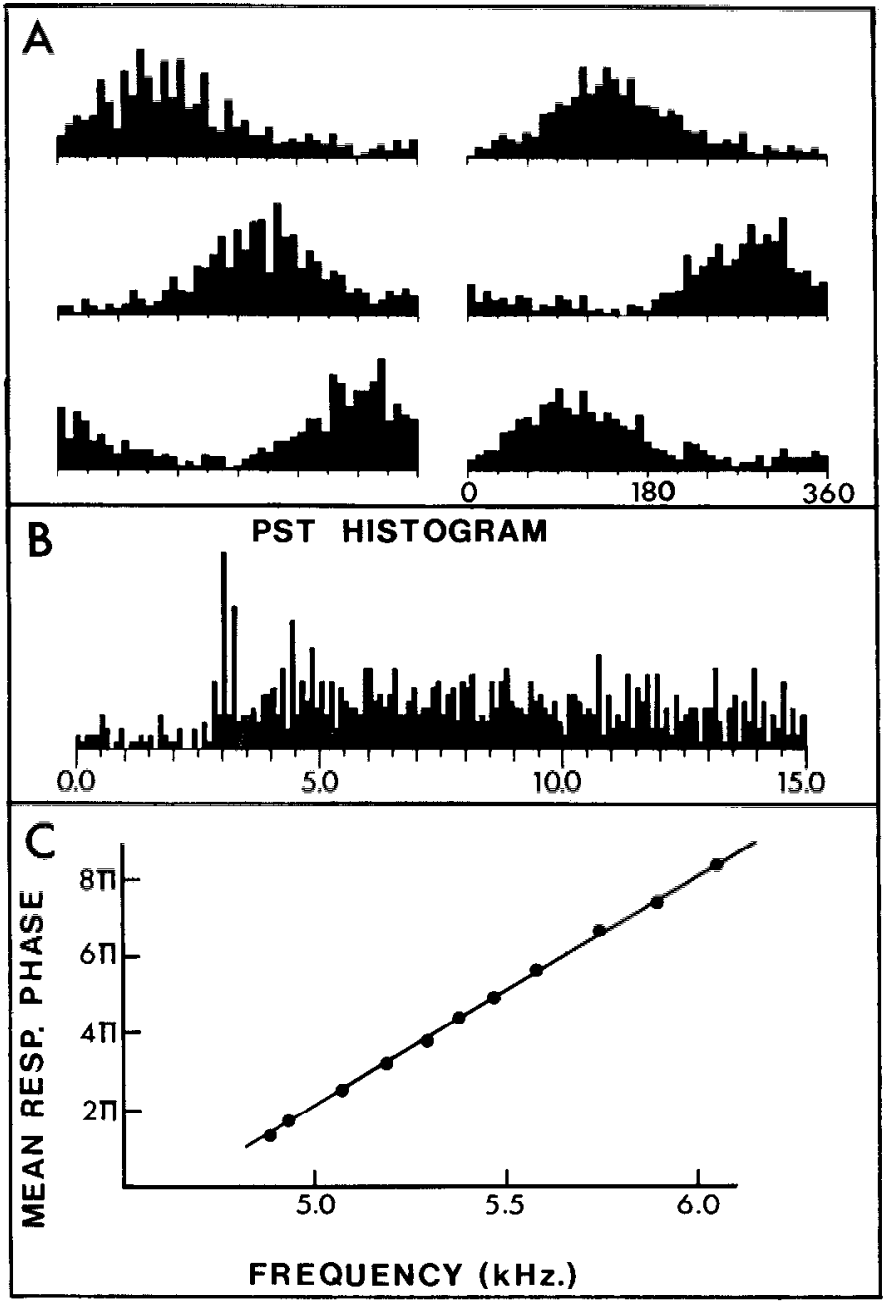

Figure 3. Demonstration of the shift in response phase with stimulus frequency; unit 3806. A, Period histograms for six different stimulus frequencies, amplitude $=64 \mathrm{~dB}$ SPL. Top left, $5076 \mathrm{~Hz}$; middle left, $5181 \mathrm{~Hz}$; bottom left, $5291 \mathrm{~Hz}$; top right, $5464 \mathrm{~Hz}$; middle right, $5586 \mathrm{~Hz}$; bottom right, $5747 \mathrm{~Hz} . B$, Post-stimulus time histogram for the response to a $5500-\mathrm{Hz}$ tone burst stimulus. Bin width is $100 \mu \mathrm{sec}$. Onset latency is approximately $2.8 \mathrm{msec}$. $C$, Graph of mean response phase as a function of stimulus frequency. For a constant delay system: $d T=d \phi / 2 \pi d F$. For these data, $d T$ computes as $2.96 \mathrm{msec}$, in agreement with the latency measured from the PST histogram.

quencies below the best frequency, there is a progressive advance in mean response phase as intensity decreases. (3) For frequencies above the best frequency, there is a progressive delay in mean response phase as intensity decreases. The effects of intensity on phase locking as a function of frequency for a typical unit are shown in Figure 4. Period histograms for the response to frequencies below, near, and above the unit's best frequency at four different intensities are shown at the top of the figure. A graph of mean response phase as a function of frequency at four different intensities is shown at the bottom of the figure. The slope of the function decreases with increasing intensity, and all of the lines intersect at a common point. The point at which the lines intersect (i.e., no phase change with intensity) corresponds to the best frequency of the unit. At frequencies away from this point, the rate of change of phase with intensity (distance between the separate lines) increases. The direction of the phase change depends on whether the frequency is higher or lower than the best frequency. A phase shift at frequencies lower than the best frequency is balanced by an opposite phase shift at frequencies higher than the best frequency. The size of the phase shift was no more than $200 \mu \mathrm{sec}$ for frequencies above $2 \mathrm{kHz}$. In the case illustrated, the largest shift in response phase observed corresponds to a $132.2-\mu$ sec time advance at $2850 \mathrm{~Hz}$ (below best frequency) and a 56.4- $\mu$ sec time delay at 3940 $\mathrm{Hz}$ (above best frequency) for a stimulus attenuation of $30 \mathrm{~dB}$.

Phase locking in the angular nucleus. The angular nucleus is anatomically distinct from the magnocellular nucleus. Figure 5 shows cross-sections through the owl's brainstem depicting the locations of the magnocellular and angular cochlear nuclei, the incoming eighth nerve

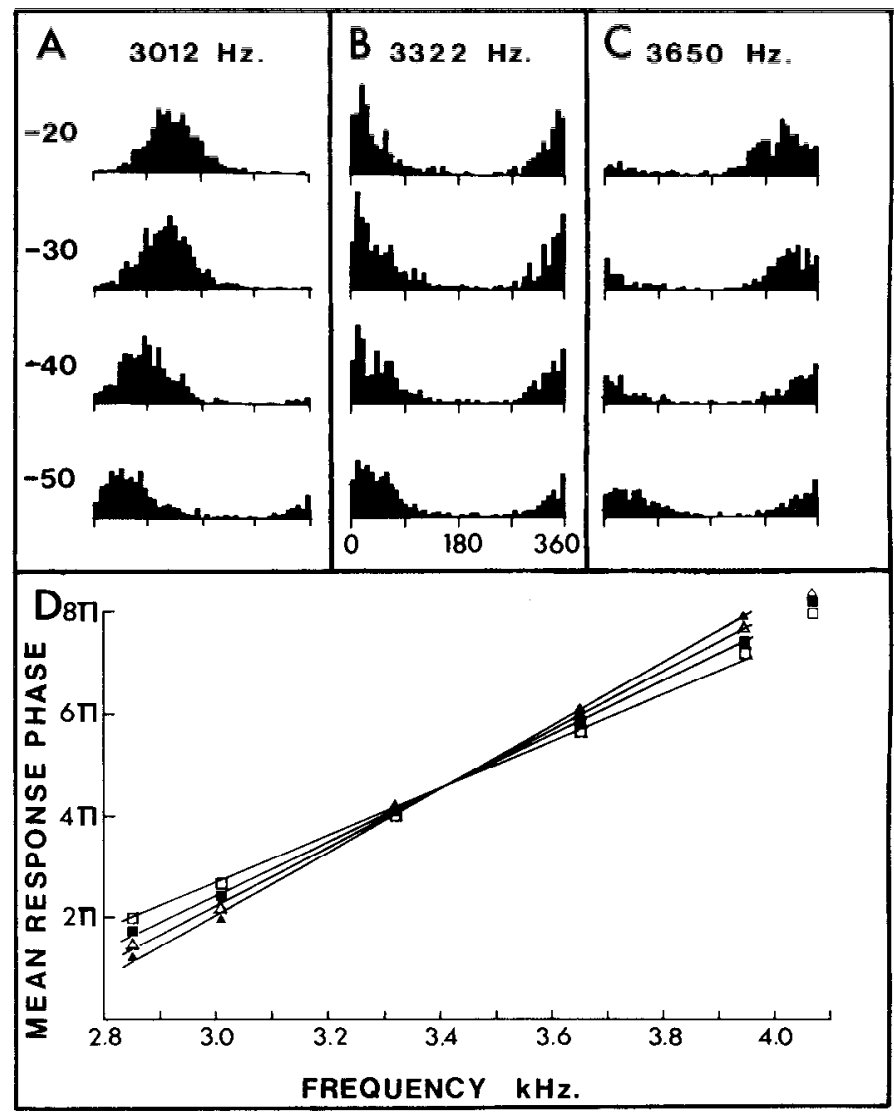

Figure 4. Phase locking in a magnocellular neuron as a function of both frequency and intensity; $0 \mathrm{~dB}=88 \mathrm{~dB}$ SPL. $A$, Period histograms at three different frequencies, each at four different intensities. Left, frequency $=3012 \mathrm{~Hz}$ (below the unit's best frequency); mean response phase decreases from $132.5^{\circ}$ at $-20 \mathrm{~dB}$ to $51.8^{\circ}$ at $-50 \mathrm{~dB}$. Center, frequency $=3322$ $\mathrm{Hz}$ (near the unit's best frequency); mean response phase changes from $12.7^{\circ}$ at $-20 \mathrm{~dB}$ to $28.2^{\circ}$ at $-50 \mathrm{~dB}$. Right, frequency $=3650 \mathrm{~Hz}$ (above the unit's best frequency); mean response phase increases from $305^{\circ}$ at $-20 \mathrm{~dB}$ to $377^{\circ}$ at -50 $\mathrm{dB}, B$, Plot of mean response phase as a function of stimulus frequency for the four stimulus intensities. Open squares, -20 $\mathrm{dB}$; solid squares, $-30 \mathrm{~dB}$; open triangles, $-40 \mathrm{~dB}$; solid triangles, $-50 \mathrm{~dB}$. 


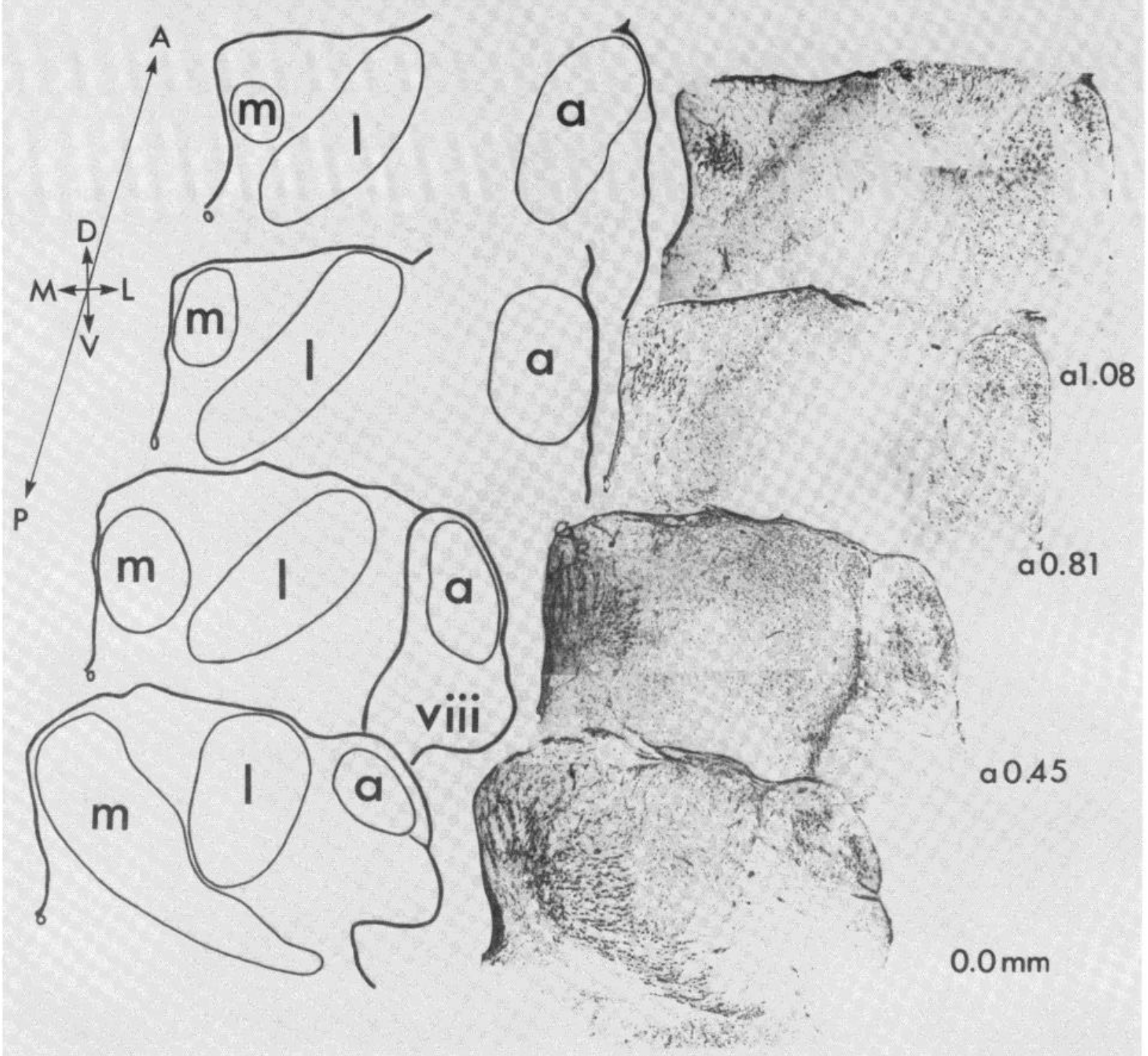

Figure 5. Right, Photomicrographs of four cresyl violet-stained coronal sections through the barn owl's brainstem at the level of the cochlear nucleus. The left edge of each section is at the midline. The most posterior section is at the bottom and the most anterior at the top. The numbers to the right of each section indicate the anterior distance in millimeters from the most posterior section. Left, Line drawings showing the locations of $\mathrm{n}$. magnocellularis $(m), \mathrm{n}$. laminaris $(l)$ (including its neuropil, which is the two most posterior sections is the only portion seen), n. angularis (a), and the acoustic nerve (viii).

fibers, and the binaural nucleus laminaris. The angular nucleus is located far lateral to the magnocellular nucleus, the binaural nucleus laminaris partially separating the two.

We used several operational criteria to distinguish the angular nucleus physiologically. These are the presence of a tonotopic order in which a unit's best frequency changes from high to low frequency as the electrode is advanced from dorsal to ventral (Konishi, 1970) and of large, easily isolated spikes. The period histograms obtained from unit responses in this region were always flat or poorly modulated, indicating that the timing of the spike discharges was independent of the phase of the stimulus. In some cases, a small peak was seen in the period histogram. These peaks were often due to random events, since they were not seen at the same phase when the same stimulus frequency was repeated. However, about half of the angular units tuned to low frequencies $(<3.5 \mathrm{kHz})$ showed weak phase locking which can be distinguished from non-phase locking by the reproducibility of the mean response phase and by an appropriate shift in mean response phase with changes in stimulus frequency.

After encountering the angular nucleus, further increases in electrode depth were often associated with a change in the quality of the action potentials from a slow or large spike to a faster wave shape, which had a distinctly different sound in the audio monitor. At this point, the period histograms shifted from flat to well modulated period histograms with high vector strengths similar to those observed in the magnocellular nucleus. Figure 6 shows an example of a large biphasic spike 
(thought to be characteristic of recordings from cell bodies), which was almost exclusively associated with flat or poorly modulated period histograms, and an example of a "fast" monophasic apike (thought to be related to fibers) associated with well modulated "phase-locked" period histograms. Our interpretation of these observations is that the flat histograms were obtained from angular neurons, whereas the phase-locked responses came from auditory nerve fibers. This view is based on the order in which the response types were encountered. There was rarely any rapid alteration or mixing of the two types. (There was some, which may be accounted for by the penetration of auditory nerve fibers into the nucleus where they make their synaptic contacts.) Phaselocked cells were usually encountered before or after a series of non-phase-sensitive cells was recorded, reflecting the anatomical relationship between the angular nucleus and the auditory nerve.

To demonstrate that the different classes of neurons encountered in penetrations through the angular nucleus represent angular neurons and auditory nerve fibers rather than two types of angular neurons, we placed lesions in phase-sensitive regions. The results of these experiments strongly support the interpretation that phase-locked responses are attributable to auditory nerve fibers. In the case shown in Figure 7, phase-locked responses were observed both before and after a series of non-phase-sensitive cells was encountered. Lesions placed at the sites where phase locking was observed are seen to straddle the angular nucleus dorsomedially and ventrally. The ventral lesion is clearly in the auditory nerve bundle. The dorsal lesion is at the edge of the nucleus in a region with a low concentration of neuronal cell bodies. The correlation of phase locking with lesions in areas containing large concentrations of fibers was seen in four other cases.

A total of 179 angular neuronons and 37 aúditory nerve fibers were isolated. Figure 8 shows a graph of vector strength as a function of unit best frequency for the two classes. Note that there is little overlap between the two populations. At frequencies greater than $3.5 \mathrm{kHz}$, no angular neurons show sensitivity to phase. Below $3 \mathrm{kHz}$, a substantial proportion of the angular neurons respond at random with respect to phase, whereas others show moderate levels of phase locking. Even so, the degree of phase locking seen in these cells is well below that observed in magnocellular units and auditory nerve fibers tuned to the same frequencies.

The data from auditory nerve fibers allow us to make some comparisons between phase locking in primary and secondary auditory neurons. It is clear that the phaselocking strength of magnocellular neurons is at least as high as that of auditory nerve fibers. In fact, for every frequency at which we recorded phase locking in the auditory nerve, there are examples of magnocellular responses which show stronger phase locking. A comparison of the two vector strength distributions shows that the distribution of auditory nerve vector strengths occupies the lower half of the magnocellular distribution. From this it would appear that phase locking improves rather than deteriorates in the owl's cochlear nucleus.

\section{Sensitivity to stimulus intensity}

General considerations. The task of studying the neural representation of stimulus intensity is complicated because there is no single response parameter such as phase

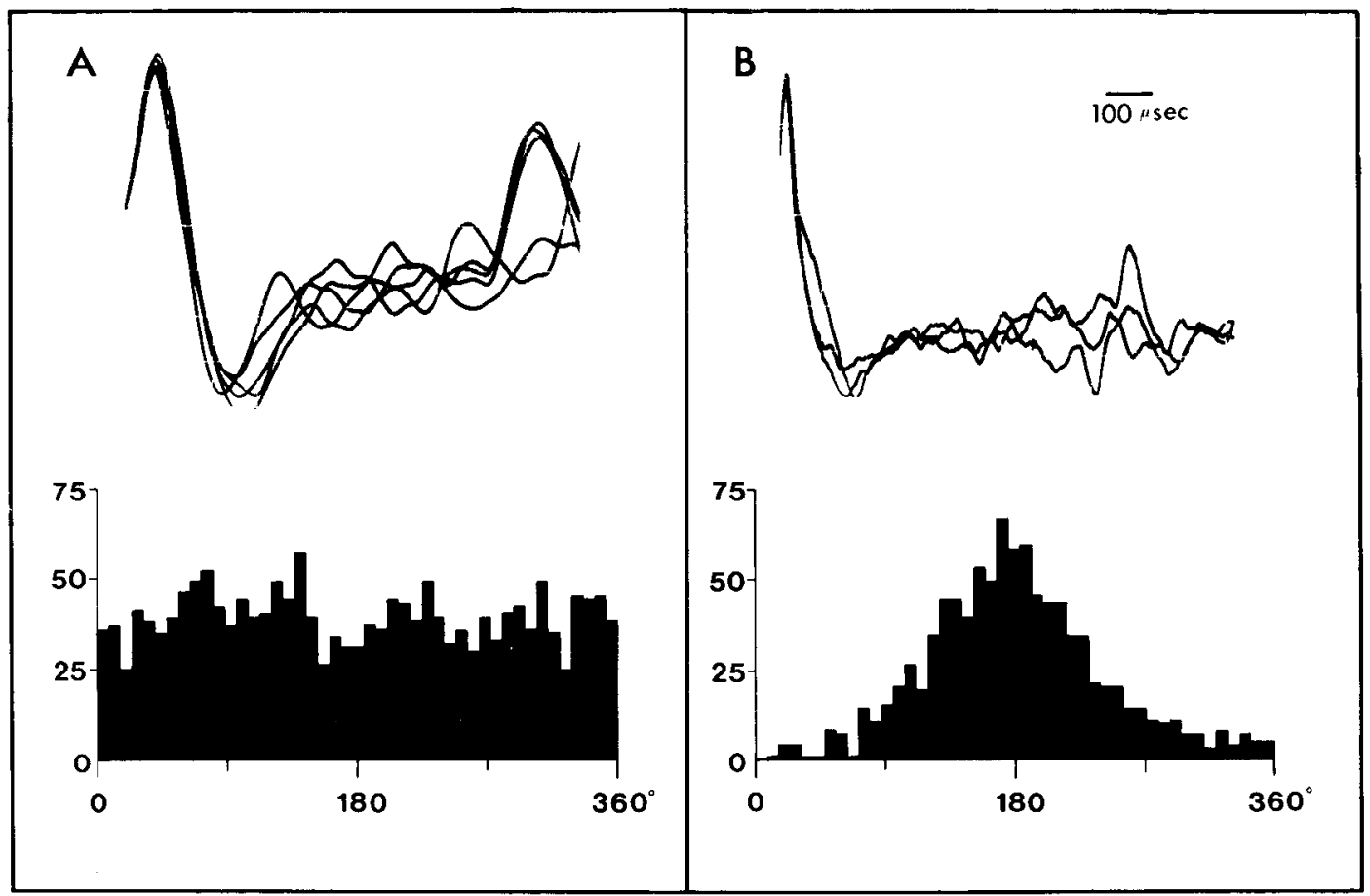

Figure 6. Extracellular recordings of spike discharges and related period histograms from penetrations through the angular nucleus. A, Above: Large spike shape tyically encountered in the tonotopic area. Below: Flat period histogram showing that spike discharges occur with equal probability at all sine wave phase angles. Stimulus frequency $=2000 \mathrm{~Hz} ; r=0.030 . B, A b o v e:$ Fast spike which was usually associated with phase-locked responses. Below, Stimulus frequency $=3225 \mathrm{~Hz} ; r=0.569$. The bar in the upper right represents a $100-\mu$ sec scale. 


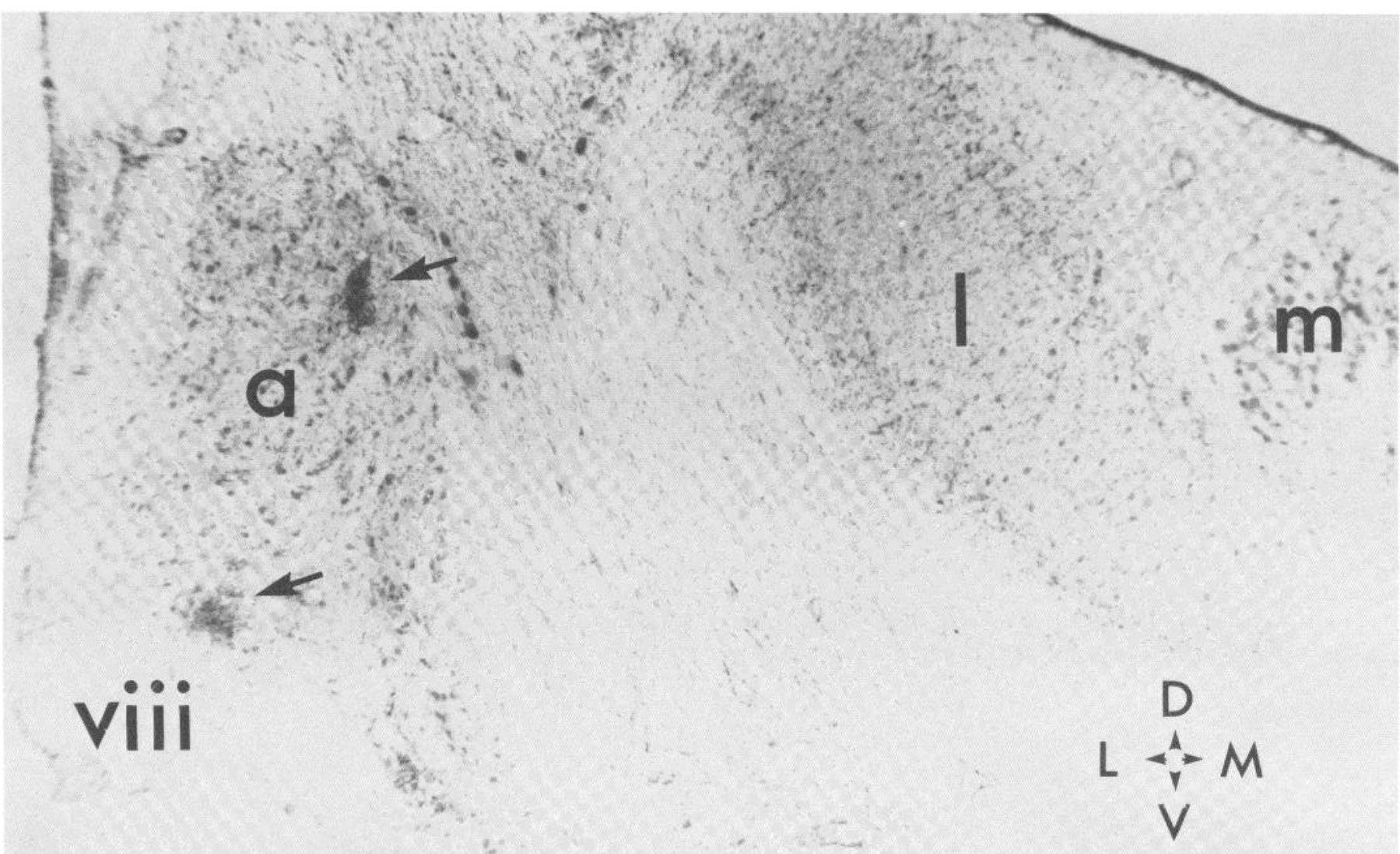

Figure 7. Photomicrograph of the brainstem from an animal in which two lesions were placed in phase-sensitive areas during a penetration through the angular nucleus. The position of each lesion is depicted by an arrow. $a$, angularis; $l$, laminaris; $m$, magnocellularis; viii, acoustic or eighth nerve.

locking that can be used to test for a neuron's sensitivity to stimulus intensity. There are several response parameters which are known to vary with intensity, and any one of them could contribute to the neural code. For simplicity, we will consider the three most prominent: spike counts, spike discharge rate, and response latency.

The response latency of auditory neurons is known to be a monotonically decreasing function of intensity (Hind et al., 1963; Aitkin et al., 1970). The latency variation occurs over a wide dynamic range so that response latency continues to decrease with increased intensity even after spike counts have reached saturation levels. This property makes response latency an attractive candidate for carrying intensity information. However, if intensity is coded by response timing, binaural neurons sensitive to interaural intensity differences should also be sensitive to interaural time differences. The barn owl's binaural neurons do not show such a time intensity trade-off for sounds of moderate duration, making it unlikely that monaural intensity is coded in the timing of the response (Moiseff and Konishi, 1981).

The number of spikes per stimulus is a function both of spike discharge rate and response duration. All of the units that we recorded in the cochlear nucleus responded in a sustained fashion to sounds of moderate duration. Some units showed a prominent burst at the beginning of the response; others did not. We did not see any consistent variation in response duration as a function of stimulus intensity. Average spike counts and average

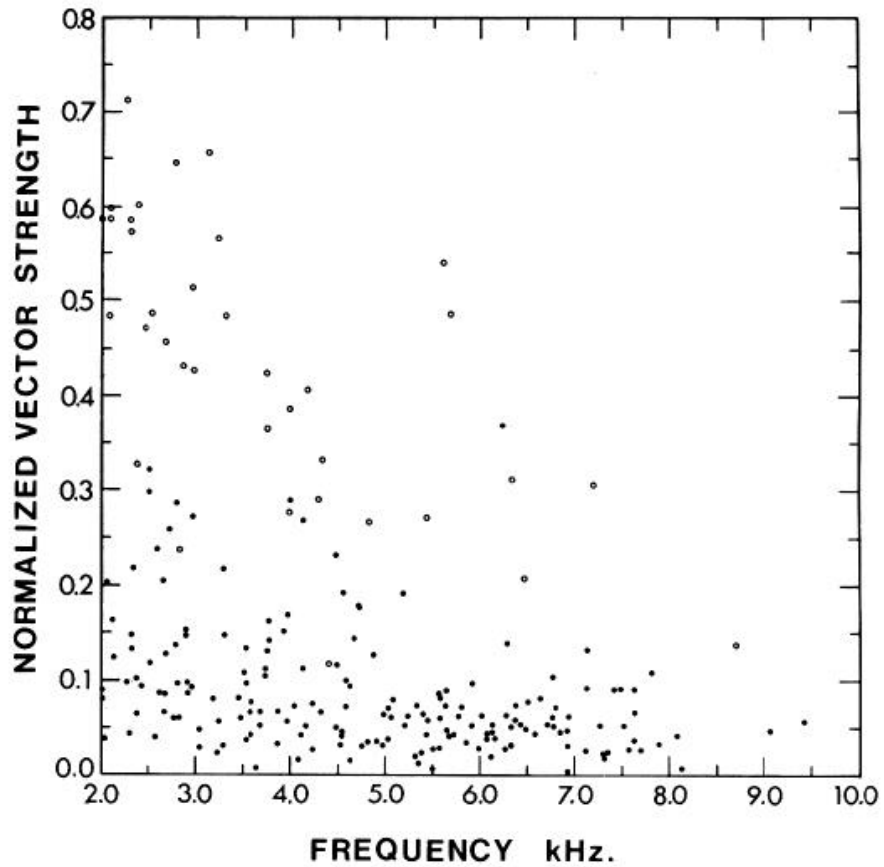

Figure 8. Vector strength as a function of best frequency plotted for 179 angular neurons (solid circles) and 37 acoustic nerve fibers (open circles).

spike discharge rate (the reciprocal of the mean spike interval) appeared to change in parallel as intensity was varied, indicating that the two parameters are roughly 
equivalent. For this reason, we focused our attention on average spike counts as a function of intensity. Intensityspike count functions were obtained using pure tone bursts of $100 \mathrm{msec}$ in duration, with the frequency at or near the best frequency of the neuron.

The spike count functions of all of the units we recorded in the cochlear nucleus were of the classical monotonic form. Several criteria were used to assess how well a neuron's response reflects changes in stimulus intensity. These are: (1) the dynamic range of the response, (2) the sensitivity in terms of change of spike counts with intensity, and (3) the variability of spike counts at a given intensity from one stimulus presentation to the next.

Magnocellular nucleus. Intensity spike count functions were obtained from 38 magnocellular units. These units had high rates of spontaneous activity. Their intensity spike count functions were characterized by a small increase in spikes over background levels from threshold to saturating intensities and by a narrow dynamic range of sensitivity to intensity changes (usually $20 \mathrm{~dB}$ or less). Figure 9 shows several examples of magnocellular spike count functions.

While the response of most magnocellular units changes moderately with changes in intensity, a few units responded at approximately the same rate for every intensity that was tested (see example Fig. 9D). Period histograms showed that such units were actually responding to the stimulus. In these and other units, phase locking was observed at intensities where average spike counts are indistinguishable from spontaneous levels, indicating that a stimulus can influence spike timing without affecting spike number. In this case, spikes encode temporal information but convey little or no information about stimulus intensity. This lack of sensitivity to stimulus intensity may be due to the neuron's inherent spontaneous activity. The auditory nerve input may determine the timing of the spikes without affecting their average rate.

Angular nucleus. The intensity spike count functions of angular neurons were clearly different from those of magnocellular units. They were characterized by low rates of spontaneous discharge, large increases in spike number with intensity, and wide dynamic ranges (usually greater than $20 \mathrm{~dB}$, often $30 \mathrm{~dB}$ or more). Several examples of angular spike count functions are shown in Figure 10.

Figure 11 shows distributions of several response properties for both magnocellular and angular neurons. As a population, angular units had significantly greater sensitivity (spikes per decibel) and dynamic range. This was also expressed in spontaneous levels compared to saturation levels. Angular units have lower spontaneous and higher saturation levels than magnocellular units. In all of these comparisons, the two populations were significantly different (the Student's $t$ test, $p<0.0005$ ).

To compare response variability, we calculated the percentage of the average spike number represented by the spike number standard deviation for magnocellular and angular neurons responding at a given level. A response of 20 to 30 spikes/stimulus was chosen because most units were responding above spontaneous levels at this rate, and almost all of the neurons responded with this rate at some intensity (although for many magnocellular units, this represents saturation level). The distributions (Fig. $11 F$ ) reveal that angular neurons had less variability in their response than did magnocellular neurons at similar discharge levels. The difference between the two distributions was significant $(p<0.0005)$.

Response variability was also examined by analyzing

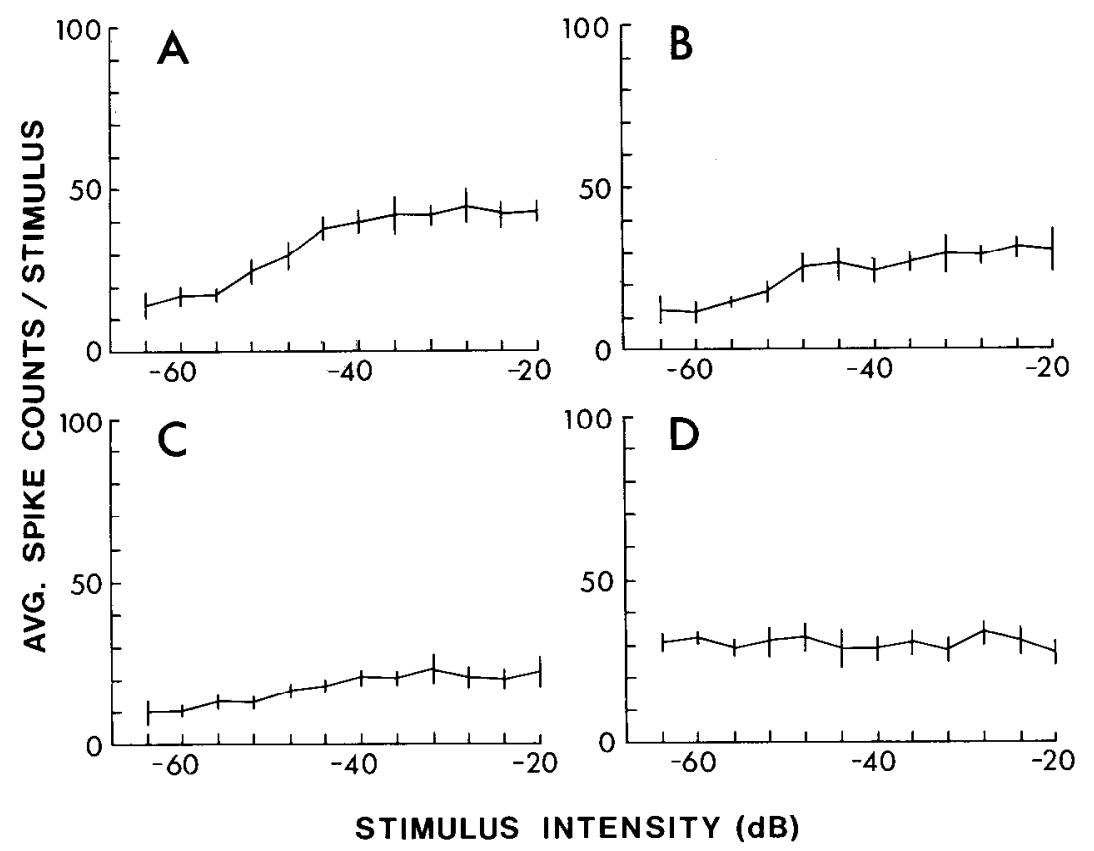

Figure 9. Intensity-spike functions for four magnocellular neurons. $A$ to $D$ show representative functions from the largest change in spike counts with intensity $(A)$ to the smallest $(D)$. In $D$, spike counts for all intensities were similar to spontaneous levels. Period histograms showed that the neuron was actually responding to the stimulus. 


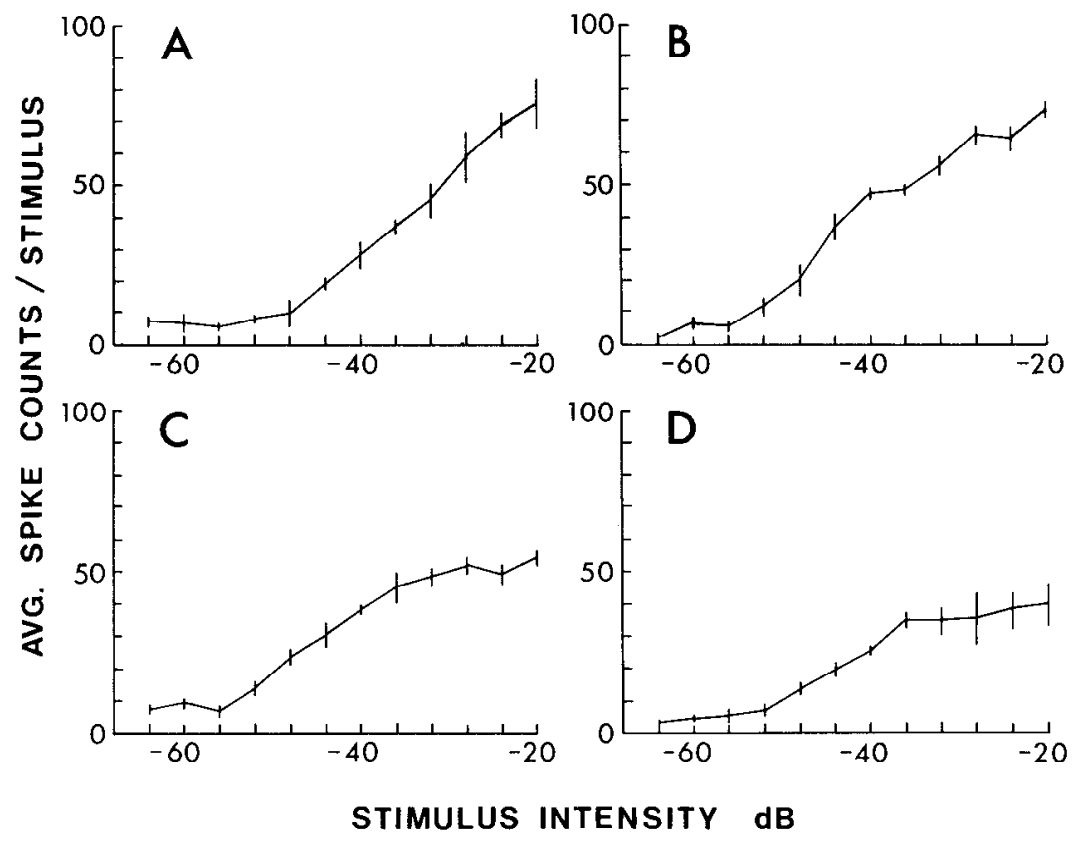

Figure 10. Intensity-spike count functions for four different angular neurons. $A$ to $D$ represent the range of spike count functions observed. Note that $D$ (showing relatively small change in spike counts with intensity) is similar to Figure $9 A$, which is one of the most intensity-sensitive magnocellular responses.

spike discharge regularity. One method is to calculate the coefficient of variation for the spike interval distribution of a neuron's discharge. This is the ratio of the standard deviation of spike intervals to the mean interval. Neurons with regular discharge patterns have low coefficients of variation. We measured the coefficient of variation for the response of 66 angular and 55 magnocellular neurons. Eighty-five percent of the angular neurons (56 of 66) had coefficients of variation of less than 0.65 , whereas $87 \%$ of the magnocellular neurons ( 48 of 55) had values greater than this. The distributions for the two populations were normal and were significantly different $(p<0.0005)$.

\section{Frequency tuning}

From signal processing theory, one would expect that the temporal resolution of a magnocellular neuron's response is achieved at the expense of frequency resolution. However, it is important to obtain phase comparisons at many different frequencies to achieve an unambiguous determination of azimuth (see "Discussion"). Therefore, we compared the frequency-tuning sharpness of magnocellular neurons with that of angular neurons in which there is no evidence of sensitivity to stimulus timing. Figure 12 shows several representative frequency-tuning curves obtained from neurons in the angular and magnocellular nuclei. Frequency-tuning sharpness was assessed by measuring Q10, which is equal to tuning curve width at $10 \mathrm{~dB}$ above absolute threshold divided by the characteristic frequency. We found that there was essentially no difference between the Q10 values for magnocellular and angular neurons. Magnocellular neurons had Q10 values from 2.3 to 8.75 for characteristic frequencies from 1.9 to $8.8 \mathrm{kHz}$. Angular neurons had Q10s ranging from 2.4 to 8.2 , over the same frequency range. If anything, the tuning curves of magnocellular neurons are somewhat sharper than those of angular neurons. This indicates that the auditory system is able simultaneously to preserve temporal and frequency resolution.

\section{Discussion}

High frequency phase locking in the barn owl's cochlear nucleus. Our results show that magnocellular neurons respond in a phase-locked manner at frequencies at least as high as $9.0 \mathrm{kHz}$. The magnocellular nucleus is thought to be homologous to the mammalian anteroventral cochlear nucleus where phase-locked responses are also observed (Lavine, 1971; Goldberg and Brownell, 1973; Rose et al., 1974). However, the maximum frequencies to which magnocellular units show phase locking is about an octave higher than the upper limit for phase locking which has been described for units in the mammalian auditory nerve and cochlear nucleus (Rose et al., 1967; Anderson et al., 1971; Johnson, 1974). Although these results do not imply that similar phase locking should have been observed in mammals, they do suggest that the limit of 4 to $5 \mathrm{kHz}$ previously described is not an absolute limit for all auditory systems. It follows that any predictions based on such a limit must be re-evaluated by direct experimental observation of the animal in question.

Intracellular recordings from mammalian cochlear inner hair cells have revealed the presence of an $\mathrm{AC}$ component of the receptor potential in response to low frequency stimuli (Russell and Sellick, 1978). The size of the $\mathrm{AC}$ component declines with increased frequency at about the same rate that phase locking disappears in auditory nerve discharges. This suggested that phase 


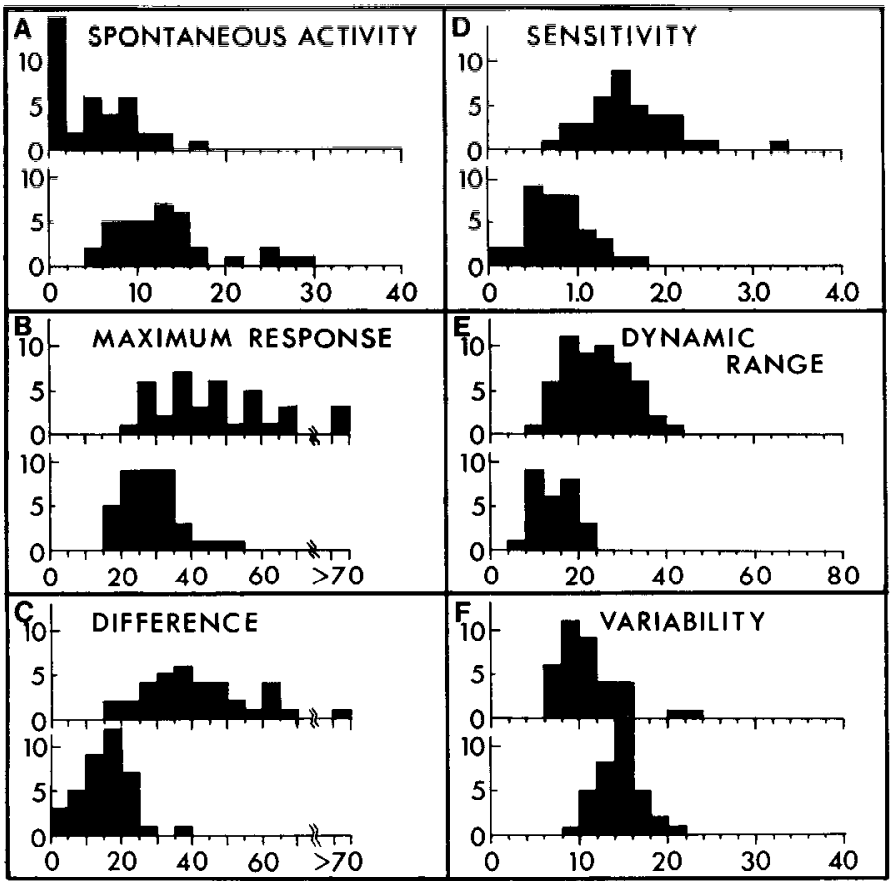

Figure 11. Histograms of response properties derived from spike count functions of angular and magnocellular neurons. In each case, histograms for the angular nucleus population are shown at the top. $A$, Spontaneous activity in spikes per 150 msec (nucleus angularis, $n=38$, mean $=6.21 \pm 4.36$; nucleus magnocellularis, $n=38$, mean $=14.16 \pm 5.85$ ) $B$, Maximum response or saturation level (spikes per stimulus) (n. angularis, $n=38$, mean $=48.68 \pm 14.96$; n. magnocellularis, $n=38$, mean $=32.63 \pm 11.19)$. $C$, Difference between saturation and spontaneous levels (spikes per $150 \mathrm{msec}$ ) (n. angularis, $n=38$, mean $=44.03 \pm 14.68 ; \mathrm{n}$. magnocellularis, $n=38$, mean $=18.03 \pm$ 7.22). $D$, Response sensitivity (in terms of change in spike counts per decibel) within the dynamic range of the response (n. angularis, $n=38$, mean $=1.64 \pm 0.5$; $\mathrm{n}$. magnocellularis, $n$ $=38$, mean $=0.82 \pm 0.36$ ). $E$, Dynamic range (in decibels) from spontaneous to saturation level ( $\mathrm{n}$. angularis, $n=54$, mean $=$ $26.30 \pm 7.36$; n. magnocellularis $n=27$, mean $=16.44 \pm 4.48$ ). $F$, Variability in the response (percentage of mean spike counts represented by the standard deviation) for spike count levels from 20 to 30 spikes/stimulus $(\sim 200$ to 300 spikes/sec) (n. angularis, $n=36$, mean $=12.00 \pm 3.64$; n. magnocellularis, $n$ $=36$, mean $=15.50 \pm 2.55$ ).

locking is a consequence of the $\mathrm{AC}$ component of the receptor potential.

In the barn owl, the rate at which phase locking declines with frequency is slower than in mammals, resulting in an extension in the frequency range over which phase locking is observed. It will be of interest to see if this increased frequency range is accompanied by an increased range over which an $\mathrm{AC}$ component to the hair cell receptor potential is observed. If so, differences in membrane properties between mammalian and barn owl hair cells, or differences in the mechanical properties of the basilar membranes may help to explain the observed discrepancy in phase-locking ability.

Separation of phase and intensity coding in the cochlear nucleus. The results described in this paper suggest that there is a separation of phase and intensity coding at the level of the cochlear nucleus. The magnocellular nucleus contains neurons which respond most often at a particular phase of the stimulus, thereby preserving information on waveform timing in the temporal pattern of their response. The response of these neurons does not appear to be greatly dependent on the intensity of the stimulus. This is true for both the temporal pattern (phase locking) and to some extent the overall number of spikes generated by the stimulus. In the angular nucleus, neurons respond at random with respect to stimulus phase, the timing of the spike discharges bearing little or no relation to the phase of the stimulus. The spike count functions of angular neurons have higher dynamic ranges, higher changes in spike counts with intensity, and lower variabilities than those of magnocellular neurons, implying that angular neurons have a greater capacity to carry information about stimulus intensity. In summary, magnocellular neurons show sensitivity to phase and not to intensity. The reverse is true for angular neurons. This supports the idea that each nucleus is specialized to process one acoustic parameter and not the other. This is the first example of a functional distinction between two anatomically distinct components of the cochlear nucleus. The general implication is that different subnuclei of a sensory nucleus receiving the same inputs have different functional roles.

Separate functional roles may eventually be defined in cochlear nucleus subdivisions in other species. This is indicated by several similarities between our results and those of other workers. In the redwing blackbird, Sachs
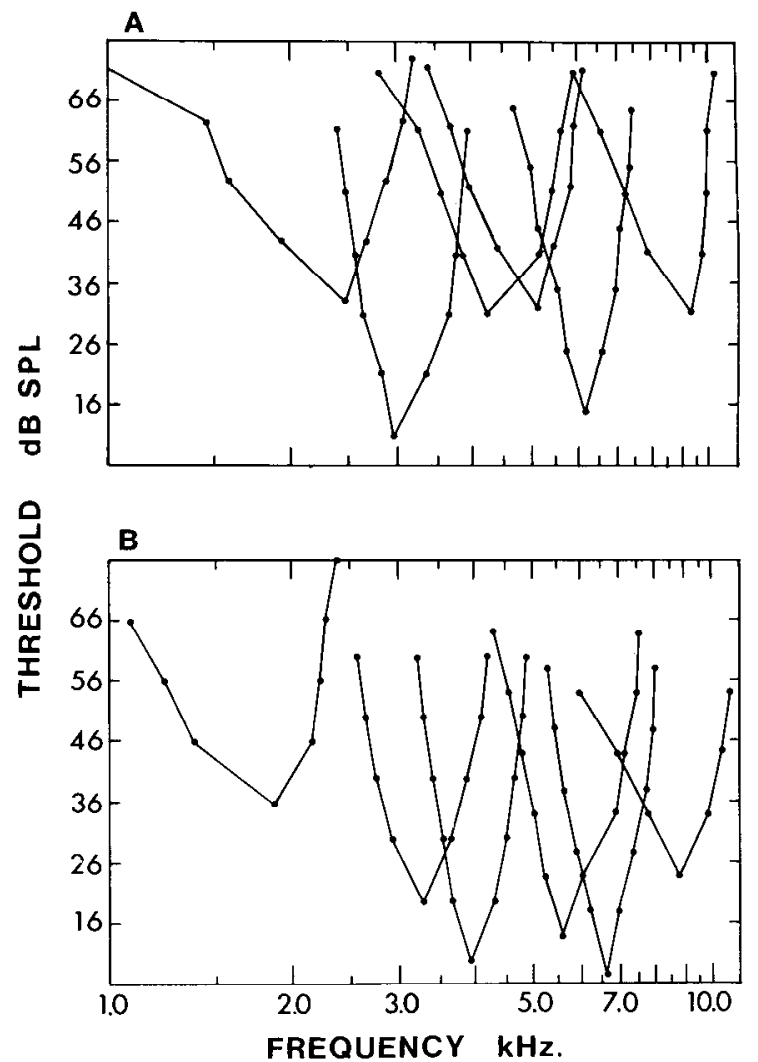

Figure 12. Frequency tuning curves for angular $(A)$ and magnocellular $(B)$ neurons. Threshold values are in decibels. re 0.0002 dyne $/ \mathrm{cm}^{2}$. 
and Sinnott (1978) found similar differences in sponta= neous activity and discharge regularity between angular and magnocellular neurons, i.e.., mágnocellular neurons had more spontaneous activity and less regular discharge patterns than did angular neurons. These authors also reported that all of the units they recorded in the magnocellular nucleus and the great majority of units in the angular nucleus had monotonic intensity spike count functions. This is similar to our result with the exception that we did not find a small percentage $(10 \%)$ of nonmonotonic units in the angular nucleus as did Sachs and Sinnott.

Differences in the response properties of neurons in different cochlear nuclei could either be due to the innervation of each nucleus by auditory nerve fibers with different response properties or to differences in the synaptic contacts between auditory nerve fibers and cochlear nucleus neurons. There is some evidence to suggest that the latter hypothesis is correct. First, primary auditory fibers are known to divide into two collaterals, one innervating the magnocellular nucleus and the other innervating the angular nucleus (Boord and Rasmussen, 1963). This is a commonly observed phenomenon, and is likely that most if not all auditory nerve fibers send a branch to each nucleus. Secondly, the morphology of afferent synapses appears to be different in the angular and magnocellular nuclei. In the angular nucleus, numerous small varicosities in the afferent processes are seen (T. Takahashi, personal communication), and it is likely that synapses in the angular nucleus are spread out onto the dendrites of postsynaptic cells. In the magnocellular nucleus, afferent endings form calyces resembling the end bulbs of Held described in the mammalian anteroventral cochlear nucleus (Jhaveri and Morest, 1982). Magnocellular neurons do not have prominent dendrites.

The direct contact of afferent synapses onto the somata of magnocellular neurons (where the postsynaptic action potential is generated) should result in a faithful temporal synchrony of input and output spikes (Pfeiffer, 1966; Molnar and Pfeiffer, 1968; Hackett et al., 1982). By spreading the synaptic contacts along the length of dendrites as in the angular nucleus, the arrival of excitatory postsynaptic potentials at the cell soma will not be synchronous. If the spread of input timing is great enough, information about stimulus phase will be lost. Some low frequency angular neurons had weak phase locking, suggesting that the loss of phase sensitivity is incomplete at low frequencies. The spatial spread of the synaptic input could enable a more temporally sustained postsynaptic potential to be generated so that the synaptic input from consecutive spikes can be added together. In this way, the average level of the postsynaptic potential will be related to the rate of synaptic input. Intensity sensitivity could result from the recruitment and rate coding of several afferent fibers converging on a single angular neuron. This model would also explain the low spontaneous rate of angular neurons, since infrequent inputs would not be able to summate (due to decay of the postsynaptic potential generated by each input spike) to induce postsynaptic spikes.

Thus, is it likely that a clear correlation can be estab- lished between a physiological distinction in response properties and a morphological difference in synaptic contacts. That the same auditory nerve fiber can form synapses with completely different morphologies on different collateral branches raises some interesting developmental questions.

The results discussed to this point indicate that each nucleus is sensitive to phase or to intensity but not to both. Our results also indicate that the processing of one parameter is not affected by changes in the other. In the angular nucleus, intensity coding is already independent of phase coding due to the lack of phase information in the response of angular neurons. In the magnocellular nucleus, the mean phase of a unit's response is found to be constant over a wide range of intensities, but only at the neuron's best frequency. However, phase shifts with intensity changes on either side of the best frequency (in units of time) are small relative to changes in onset latency. Furthermore, phase shifts at frequencies lower than the best frequency are balanced by equal and opposite phase shifts at frequencies above the best frequency (similar results have been reported for the auditory nerve of squirrel monkeys by Anderson et al., 1971). The initial binaural comparison of phase information in n. laminaris (Moiseff and Konishi, 1983) is made between neurons with the same best frequencies (Parks and Rubel, 1975). Therefore, changes in average intensity would cause the same phase shifts to occur on each side, resulting in no net effect on the binaural phase difference. That is, the system would be immune to changes in the loudness of the sound source. Binaural comparison between neurons with the same best frequency would also minimize the effects of interaural intensity differences on phase comparison, especially at or near the best frequency where the response is strongest. Away from the best frequency, interaural intensity differences will cause shifts in phase comparison. However, a frequency which is above the best frequency for one binaural pair will be below best frequency for another pair, and the shifts produced will be of opposite sign for these two pairs. Comparison of inputs from a wide range of frequency channels at higher levels in the system can ensure that the correct location is determined. This model is supported by the fact that space-specific neurons in the inferior colliculus which are sensitive to both interaural phase and intensity differences (see below) are usually broadly tuned to frequency (Knudsen and Konishi, 1978).

The pattern of efferent connections that has been described for each nucleus further supports the notion of functional specialization. The magnocellular nucleus projects bilaterally to $\mathrm{n}$. laminaris, which is known to contain neurons sensitive to binaural time differences (Moiseff and Konishi, 1983). Laminaris does not receive inputs from the angular nucleus. The output of the angular nucleus innervates the lemniscal nucleus ventralis lemnisci lateralis pars posterior (VLPp) on both sides (Leibler, 1975). This nucleus contains neurons which are sensitive to binaural intensity differences but not to binaural time differences (Moiseff and Konishi, 1983). Therefore, not only is there a functional separation of the cochlear nuclei based on response properties, 
but this functional separation appears to be maintained in the efferent connections of each individual nucleus. The two pathways eventually converge so that by the level of the inferior colliculus ( $\mathrm{n}$. mesencephalis lateralis pars dorsalis or MLD), neurons are found which are sensitive to a narrow range of binaural phase and intensity differences (Moiseff and Konishi, 1981). These correspond to the "limited-field" or space-specific neurons described earlier by Knudsen and Konishi (1978).

Recent work in our laboratory by Takahashi et al. (1984) has shown conclusively that the combined selectivity to binaural phase and intensity differences of space-specific neurons is due to separate inputs originating in the angular and magnocellular nuclei. Blockade of transmission in the angular nucleus (by injection of lidocaine directly into the nucleus) affected the binaural intensity selectivity of space-specific neurons without affecting their binaural phase selectivity. Blockade of the magnocellular nucleus caused the opposite effect, loss of binaural phase selectivity with little or no change in binaural intensity selectivity.

The separation of phase and intensity processing suggests that the interpretation of monaural codes for both phase and intensity cannot be done at the same binaural integration site. Binaural phase differences are presumably conveyed by the difference in the absolute time of occurrence of spikes in each monaural channel. The relative timing of one spike to the next (interspike interval or its reciprocal spike rate) and not the absolute time of each spike may be important for intensity coding. Our results show that spike rate is a consistent and sensitive measure of stimulus intensity in angular neurons. Therefore, absolute timing of spikes is important in one case and not the other. This difference in interpretation for the initial binaural comparison and the need for subsequent sharpening of neuronal selectivity for each binaural cue may explain the requirement for separate processing of each parameter. Incompatibility of codes for different parameters of a sensory stimulus may explain the presence of parallel processing in other systems as well. That is, for each separate channel there is a separate code.

Behavioral correlates of phase and intensity coding. The proposed separation of phase and intensity coding in the cochlear nucleus reflects the owl's use of binaural phase and intensity information for sound localization in the horizontal and vertical dimensions, respectively. A barn owl responds to binaural phase differences in dichotic stimuli by turning its head to an azimuthal location. The location can be predicted from the sign and magnitude of the phase difference measured in free field (Moiseff and Konishi, 1981).

There are several lines of evidence suggesting that binaural intensity differences are interpreted as locations in elevation. First, there is a physical asymmetry in the position of the external ear openings within the facial ruff. Measurements of the directional sensitivity of the ears reveal that the left ear is more sensitive to sounds coming from below and the right ear to sounds coming from above (Payne, 1971). The difference in vertical sensitivity is most pronounced for frequencies from 4 to $9 \mathrm{kHz}$, which owls localize most accurately. Secondly, when one ear is plugged, owls make localization errors primarily in elevation (Knudsen and Konishi, 1979). The small azimuthal component to the localization error may be accounted for by a slight time shift introduced by the ear plug. This has been observed in cochlear microphonic recordings in ear-plugged owls (Knudsen et al., 1984).

The facial ruff feathers may be responsible for both the lack of change of binaural intensity difference with azimuth and for the direct relationship between binaural intensity difference and sound elevation. When the facial ruff is removed, the owl's ability to localize sounds in the vertical dimension is severely disrupted, while its ability to localize sounds along the horizon is not impaired (Knudsen and Konishi, 1979).

Thus, peripheral specializations allow interaural phase and intensity differences to be separately interpreted as horizontal and vertical spatial coordinates. We have shown that the physiological differences between neurons in the two cochlear nuclei provide a neural basis for the separate interpretation of interaural phase and intensity differences by the owl's brain.

\section{References}

Aitkin, L. M., D. J. Anderson, and J. F. Brugge (1970) Tonotopic organization and discharge characteristics of single neurons in nuclei of the lateral lemniscus of the cat. J. Neurophysiol. 33: 421-440.

Anderson, D. J., J. E. Rose, J. E. Hind, and J. F. Brugge (1971) Temporal position of discharges in single auditory nerve fibers within the cycle of a sine-wave stimulus: Frequency and intensity effects. J. Acoust. Soc. Am. 49: 1131-1139.

Boord, R. L., and G. L. Rasmussen (1963) Projection of the cochlear and lagenar nerves on the cochlear nucleus of the pigeon. J. Comp. Neurol. 120: 463-475.

Brawer, J. R., D. K. Morest, and E. C. Kane (1974) The neuronal architecture of the cochlear nucleus of the cat. J. Comp. Neurol. 155: 251-300.

Goldberg, J. M., and P. B. Brown (1969) Response of binaural neurons of dog superior olivary complex to dichotic tonal stimuli: Some physiological mechanisms of sound localization. J. Neurophysiol. 32: 613-636.

Goldberg, J. M., and W. E. Brownell (1973) Discharge characteristics of neurons in anteroventral and dorsal cochlear nuclei of cat. Brain Res. 64: 35-54.

Hackett, J. T., H. Jackson, and E. W. Rubel (1982) Synaptic excitation of the second and third order auditory neurons in the avian brain stem. Neuroscience 7: 1455-1469.

Hind, J. E., J. M. Goldberg, D. M. Greenwood, and J. E. Rose (1963) Some discharge characteristics of single neurons in the inferior colliculus of the cat. II. Timing of discharges and observations on binaural stimulation. J. Neurophysiol. 26: 321-341.

Jhaveri, S., and D. K. Morest (1982) Neuronal architecture in nucleus magnocellularis of the chicken auditory system with observations on nucleus laminaris: A light and electron microscope study. Neuroscience 7: 809-836.

Johnson, D. H. (1974) The response of single auditory-nerve fibers in the cat to single tones: Synchrony and average discharge rate. Ph.D. thesis, Department of Electrical Engineering, Massachusetts Institute of Technology, Cambridge.

Johnson, D. H. (1978) The relationship of post-stimulus time and interval histograms to the timing characteristics of spike trains. Biophys. J. 22: 413-430.

Knudsen, E. I., and M. Konishi (1978) Space and frequency are represented separately in auditory midbrain of the owl. J. Neurophysiol. 41: 870-884. 
Knudsen, E. I., and M. Konishi (1979) Mechanisms of sound localization in the barn owl. J. Comp. Physiol. 133: 13-21.

Knudsen, E. I., S. D. Esterly, and P. F. Knudsen (1984) Monaural occlusion alters sound localization during a sensitive period in the barn owl. J. Neurosci. 4: 1001-1011.

Konishi, M. (1970) Comparative neurophysiological studies of hearing and vocalization in songbirds. Z. Vergl. Physiol. 66: 257-272.

Lavine, R. A. (1971) Phase-locking in response of single neurons in cochlear nucleus of the cat to low frequency tonal stimuli. J. Neurophysiol. 34: 467-483.

Leibler, L. M. (1975) Monaural and binaural pathways in the ascending auditory system of the pigeon. Ph.D. thesis, Department of Psychology, Massachusetts Institute of Technology, Cambridge.

Lorente de No, R. (1933) Anatomy of the eighth nerve. III. General plan of structure of the primary cochlear nuclei. Laryngoscope 43: 327-350.

Moiseff, A., and M. Konishi (1981) Neuronal and behavioral sensitivity to binaural time differences in the owl. J. Neurosci. 1: 40-48.

Moiseff, A., and M. Konishi (1983) Binaural characteristics of units in the owl's brainstem auditory pathway: Precursors of restricted spatial receptive fields. J. Neurosci. 3: 2553-2562.

Molnar, C. E., and R. R. Pfeiffer (1968) Interpretations of spontaneous spike discharge patterns of neurons in the cochlear nucleus. Proc. IEEE 56: 993-1004.

Osen, K. K. (1969) Cytoarchitecture of the cochlear nuclei in the cat. J. Comp. Neurol. 136: 453-484.

Parks, T. N., and E. W. Rubel (1975) Organization and development of brain stem auditory nuclei of the chicken: Orga- nization of projections from $\mathrm{n}$. magnocellularis to $\mathrm{n}$. laminaris. J. Comp. Neurol. 164: 435-448.

Payne, R. S. (1971) Acoustic location of prey by barn owls (Tyto alba). J. Exp. Biol. 54: 535-573.

Pfeiffer, R. R. (1966) Anteroventral cochlear nucleus: Wave forms of extracellularly recorded spike potentials. Science 154: $667-668$.

Ramón Y Cajal, S. (1909) Histologie du Systeme Nerveux, Vol. 1, Instituto Ramon y Cajal, Madrid, Spain.

Rose, J. E., R. Galambos, and J. R. Hughes (1959) Microelectrode studies of the cochlear nuclei of the cat. Bull. Johns Hopkins Hosp. 104: 211-251.

Rose, J. E., J. F. Brugge, D. J. Anderson, and J. E. Hind (1967) Phase-locked response to low frequency tones in single auditory nerve fibers of the squirrel monkey. J. Neurophysiol. 30: 769-793.

Rose, J. E., L. M. Kitzes, M. M. Gibson, and J. E. Hind (1974) Observations on phase-sensitive neurons of anteroventral cochlear nucleus of the cat: Non-linearity of cochlear output, J. Neurophysiol. 37: 218-253.

Russell, I. J., and P. M. Sellick (1978) Intracellular studies of hair cells in the mammalian cochlea. J. Physiol. (Lond.) 284: 261-290.

Sachs, M. B., and J. M. Sinnott (1978) Responses to tones of single cells in nucleus magnocellularis and nucleus angularis of the redwing blackbird (Agelaius phoeniceus). J. Comp. Physiol. 126: 347-361.

Takahashi, T., A. Moiseff, and M. Konishi (1984) Time and intensity cues are processed independently in the auditory system of the owl. J. Neurosci. 4: 1781-1786. 